\title{
Decoherence-induced conductivity in the discrete 1D Anderson model: A novel approach to even-order generalized Lyapunov exponents
}

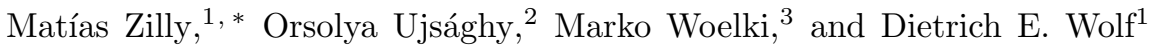 \\ ${ }^{1}$ Department of Physics, University of Duisburg-Essen and CeNIDE, 47048 Duisburg, Germany \\ ${ }^{2}$ Department of Theoretical Physics and Condensed Matter Research Group of the Hungarian Academy of Sciences, \\ Budapest University of Technology and Economics, Budafoki út 8., H-1521 Budapest, Hungary \\ ${ }^{3}$ Department of Physics, Saarland University, 66123 Saarbrücken, Germany
}

(Dated: August 27, 2021)

\begin{abstract}
A recently proposed statistical model for the effects of decoherence on electron transport manifests a decoherence-driven transition from quantum-coherent localized to ohmic behavior when applied to the one-dimensional Anderson model. Here we derive the resistivity in the ohmic case and show that the transition to localized behavior occurs when the coherence length surpasses a value which only depends on the second-order generalized Lyapunov exponent $\xi^{-1}$. We determine the exact value of $\xi^{-1}$ of an infinite system for arbitrary uncorrelated disorder and electron energy. Likewise all higher even-order generalized Lyapunov exponents can be calculated, as exemplified for fourth order. An approximation for the localization length (inverse standard Lyapunov exponent) is presented, by assuming a log-normal limiting distribution for the dimensionless conductance $T$. This approximation works well in the limit of weak disorder, with the exception of the band edges and the band center.
\end{abstract}

PACS numbers: 72.10.-d,72.15.Rn,71.23.An

\section{INTRODUCTION}

Andersor ${ }^{1}$ was the first to show that electronic eigenstates in disordered media can be localized, if the disorder exceeds a certain threshold. In the infinite, discrete one-dimensional (1D) Anderson model

$$
H=\sum_{i} \epsilon_{i}|i\rangle\langle i|+t \sum_{i}[|i\rangle\langle i+1|+\text { h.c. }]
$$

without correlations, where the onsite energies $\epsilon_{i}$ are independently distributed according to a probability density $w(\epsilon)$ (with mean value 0 , variance $\sigma^{2}$, third and fourth moments $\nu^{3}$ and $\kappa^{4}$ ), all eigenstates are localized for $\sigma^{2}>0$, i.e. the eigenfunctions decay exponentially in space. To simplify the notation we take $t=1$ as energy unit and the lattice spacing $a=1$ as length unit in the following.

Even fifty years after its foundations, the physics of Anderson localization is a very active field of research, stimulated by the recent observations of localization of light and cold atoms, cf. Refs. 2 4 and references therein.

The "absence of diffusion" due to disorder leads to an exponential increase of the electrical resistance of the localized system, if the electrons behave coherently 115 Ohmic behavior, a resistance increase proportional to the linear extension of the device, can be achieved in spite of localization, if sufficient decoherence is included $\frac{6}{6} \mathrm{In}$ recent publications we have demonstrated this using a statistical model for the effects of decoherence ${ }^{[7]}$ Also the experimental observation of sequence-dependent conduc-

\footnotetext{
*Electronic address: matias.zilly@uni-due.de
}

tance in DNA molecules (ohmic vs. exponential) 10 could be explained!11

In this article we examine the decoherence-induced conductivity in the Anderson model subjected to the phenomenological model for decoherence.

Decoherence-induced conductivity in disordered systems was already subject of various papers 12 Here we examine how much decoherence is necessary in order to obtain finite conductivity and conclude that whenever the coherence length $L_{\phi}$ fulfills

$$
L_{\phi}<\frac{1}{1-\exp \left(-\xi^{-1}\right)},
$$

ohmic conductance is reached. Here $\xi^{-1}$ is the secondorder generalized Lyapunov exponent (GLE), 15 [17 for which we report exact results for arbitrary diagonal disorder and electron energy. All higher even-order GLEs, e.g. the fourth-order GLE $\chi^{-1}$, can be calculated analytically by the same method as $\xi^{-1}$. The even-order GLEs have also been determined for nonlinear oscillators. $\frac{18}{18}$

Assuming a Gaussian limiting distribution for the logarithm of the dimensionless conductance $\ln T$, we present an approximation in which the localization length $\lambda$ (the inverse standard Lyapunov exponent LE) is determined by $\xi$ and $\chi$. This approximation works well in the limit of weak disorder with the exception of the band edges and the band center.

The paper is organized as follows. Sect. [II explains the coherent transport formalism and the phenomenological statistical model by which we include decoherence effects. In Sect. III we derive the resistivity and the limitations under which finite conductivity is found. Sect. [V] introduces the GLEs and derives $\xi^{-1}$. Using the GLEs, in Sect. $\mathrm{V}$ we perform an approximate calculation of the localization length. Sect. VI discusses the results and 
conclusions. The Appendices contain details of the calculations.

\section{MODEL}

The idea of the statistical model for the effects of decoherence on electron transport is the following. ${ }^{77} \mathrm{We}$ describe the electrons in a single-electron picture. Decoherence is modeled by stochastic events: The electron, which in general is considered coherently, is extracted and re-injected at decoherence events, which take place at random locations in space. Thereby it loses its coherence completely. The quantity of interest, e.g. the conductance $e^{7 / 9111}$ or resistance $e^{819}$ is calculated for a given decoherence configuration, a division of the system into coherent and decoherence regions. Afterwards the quantity of interest is averaged over an ensemble of decoherence configurations. Here we apply our statistical decoherence approach to the Anderson model in the following way, cf. Fig. 1. which is a slight modification of the original.

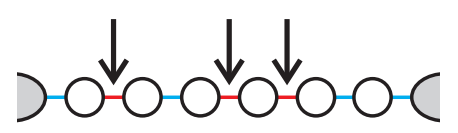

(a)

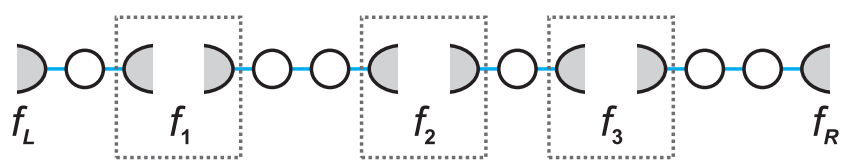

(b)

FIG. 1: (Color online) A coherent chain of $N=6$ sites coupled to wide-band contacts. (a) Three decoherence bonds introduced at random according to a probability $p$ form a decoherence configuration. (b) The resulting equivalent set of coherent chains. The $f_{k}$ denote the electron energy distribution functions at the contacts and decoherence bonds.

According to the probability $p$, the bonds of the Anderson Hamiltonian $H$ are replaced by decoherence bonds, virtual contacts in the wide-band limit which cause a purely imaginary self-energy $-i \eta$ to the neighboring regions of the system, forming a decoherence configuration. Thus $p$ and $\eta$ are the two parameters by which we describe phenomenologically the effect of decoherence.

A decoherence bond represents the environmental decohering effect, or a decoherent escape into the rest of the electronic system. An electron is extracted out of the system at the decoherence bond (the bond represents a reservoir) and re-injected with the same energy (no inelastic effects are considered) at the same bond involving a complete loss of phase coherence.

The Green's function of a coherent subsystem which is delimited by two decoherence bonds and contains the sites $|1\rangle-|j\rangle$ reads

$$
G \equiv[E-H+\mathrm{i} \eta(|1\rangle\langle 1|+| j\rangle\langle j|)]^{-1},
$$

where $H$ is the Hamiltonian restricted to the sites between the two decoherence bonds, and $E$ is the total electron energy. Due to the simple structure of the self-energies, the transmission through the subsystem according to the Nonequilibrium Green's function method (NEGF) ${ }^{6}$ reads

$$
T_{j}=4 \eta^{2}|\langle 1|G| j\rangle|^{2}
$$

and can be evaluated recursively because of the tridiagonal structure of $G^{-1}$ :

$$
\frac{1}{T_{j}}=\frac{\left|r_{j}-\eta^{2} s_{j-1}+\mathrm{i} \eta\left(r_{j-1}+s_{j}\right)\right|^{2}}{4 \eta^{2}},
$$

with the polynomials

$$
\begin{aligned}
r_{j} & =\left(E-\epsilon_{j}\right) r_{j-1}-r_{j-2}, & & s_{j}=\left(E-\epsilon_{j}\right) s_{j-1}-s_{j-2}, \\
r_{0} & =1, & & s_{1}=1, \\
r_{-1} & =0, & & s_{0}=0 .
\end{aligned}
$$

For the derivation of Eqs. (5) and (6), see the Appendix A.

Although the dimensionless conductance $T$ is often calculated without making reference to a coupling to external leads $\frac{19}{19}$ the results for large coherent systems which we discuss below do not depend on the model parameter $\eta$, as it becomes an irrelevant boundary condition (cf. Appendix C).

Under the condition of a complete loss of phase coherence without energy relaxation at the decoherence bonds, we have shown in Ref. 7, that the resistance of a decoherence configuration at infinitesimal bias is the sum of the individual subsystem resistances,

$$
R=\frac{h}{2 e^{2}} \sum_{k} \frac{1}{T_{k}},
$$

where $k$ enumerates the coherent subsystems, and $\frac{2 e^{2}}{h}=$ 1 is the quantum of conductance assuming spin degeneracy, which we take as our conductance unit. To obtain the resistance as a system parameter, $R$ is to be averaged over all decoherence configurations.

Now consider the thermodynamic limit of an infinite system. As the locations of the decoherence bonds are uncorrelated, the relative frequency of the subsystem size $j$ is $p q^{j-1}$, where

$$
q \equiv 1-p
$$

is the probability of a regular bond. On average, the subsystem size is

$$
L_{\phi}=\frac{1}{p},
$$

defining the coherence length in our model. In the infinite system, subsystems of size $j$ appear infinitely many times, contributing on average $\left\langle 1 / T_{j}\right\rangle$ to the resistance. Here, $\langle\cdot\rangle$ denotes the average over the disorder. 


\section{RESISTIVITY OF THE SYSTEM}

We define the resistivity of the system as the resistance per length in the limit of an infinite system:

$$
\rho=p^{2} \sum_{j=1}^{\infty} q^{j-1}\left\langle\frac{1}{T_{j}}\right\rangle .
$$

Naturally this definition is restricted to those values $q<q^{*}$ for which the series converges. Then the system behaves ohmically. For $q>q^{*}$, localization dominates, and the resistance increases exponentially with the system size. This is the decoherence induced conductivity: As the decoherence density $p$ increases over the critical value $p^{*}=1-q^{*}$, there is a transition from localized to ohmic behavior. $2 j$

The disorder average $\left\langle 1 / T_{j}\right\rangle$ is a polynomial of order

$$
\begin{aligned}
\left\langle\frac{1}{T_{j}}\right\rangle & \equiv \int_{-\infty}^{\infty} \mathrm{d} \epsilon_{1} \ldots \mathrm{d} \epsilon_{j} \frac{1}{T_{j}} \prod_{i=1}^{j} w\left(\epsilon_{i}\right) \\
& =\frac{1}{2}+\frac{1}{4 \eta^{2}} R_{j}+\frac{1}{2} R_{j-1}+\frac{\eta^{2}}{4} R_{j-2}
\end{aligned}
$$

where we define

$$
R_{j} \equiv \int_{-\infty}^{\infty} \mathrm{d} \epsilon_{1} \ldots \mathrm{d} \epsilon_{j} r_{j}^{2} \prod_{i=1}^{j} w\left(\epsilon_{i}\right)
$$

Using Eq. (6) we find a recursion for the $R_{j}$ :

$$
\begin{aligned}
R_{j}= & \int_{-\infty}^{\infty} \mathrm{d} \epsilon_{1} \ldots \mathrm{d} \epsilon_{j}\left[\left(E^{2}-2 E \epsilon_{j}+\epsilon_{j}^{2}\right) r_{j-1}^{2}\right. \\
& \left.\quad+2\left(\epsilon_{j}-E\right) r_{j-1} r_{j-2}+r_{j-2}^{2}\right] \prod_{i=1}^{j} w\left(\epsilon_{i}\right) \\
= & \left(E^{2}+\sigma^{2}\right) R_{j-1}-2 E S_{j-1}+R_{j-2},
\end{aligned}
$$

where

$$
S_{j} \equiv \int_{-\infty}^{\infty} \mathrm{d} \epsilon_{1} \ldots \mathrm{d} \epsilon_{j} r_{j} r_{j-1} \prod_{i=1}^{j} w\left(\epsilon_{i}\right)
$$

itself fulfills the recursion

$$
\begin{aligned}
S_{j} & =\int_{-\infty}^{\infty} \mathrm{d} \epsilon_{1} \ldots \mathrm{d} \epsilon_{j}\left[\left(E-\epsilon_{j}\right) r_{j-1}^{2}-r_{j-1} r_{j-2}\right] \prod_{i=1}^{j} w\left(\epsilon_{i}\right) \\
& =E R_{j-1}-S_{j-1} .
\end{aligned}
$$

The initial conditions $R_{-1}=0, S_{0}=0$ and $R_{0}=1$ allow to determine the disorder-averaged resistance $\left\langle 1 / T_{j}\right\rangle$ of a subsystem of size $j$ via Eqs. (13)- 15 .
Using the recursions in Eqs. 13) and 15 we calculate the generating function of the $R_{j}$ :

$$
\begin{aligned}
\mathcal{R}(z) & \equiv \sum_{j=1}^{\infty} R_{j} z^{j-1} \\
& =\frac{1}{N_{1}}\left(-z^{2}+\left(E^{2}-\sigma^{2}-1\right) z-E^{2}-\sigma^{2}\right),
\end{aligned}
$$

where

$$
N_{1} \equiv z^{3}+\left(1-E^{2}+\sigma^{2}\right) z^{2}+\left(E^{2}+\sigma^{2}-1\right) z-1 .
$$

With Eq. 11] we evaluate the resistivity $\rho$ [Eq. (10] in terms of the generating function $\mathcal{R}(z=q)$ arriving at the closed form valid for $q<q^{*}$

$$
\begin{aligned}
\rho= & \frac{p}{2}+\left[p^{2}\left(\frac{1}{2}+\frac{1}{4 \eta^{2}}\left(\sigma^{2}+E^{2}\right)\right)\right. \\
& \left.+p^{2} q\left(\frac{1}{4 \eta^{2}}+\frac{\eta^{2}}{4}-\frac{2 E^{2}}{4 \eta^{2}(1+q)}\right)\right] \\
& \times\left[1-\left(\sigma^{2}+E^{2}\right) q-q^{2}+\frac{2 E^{2} q^{2}}{1+q}\right]^{-1}
\end{aligned}
$$

Fig. 2 displays the resistivity $\rho$ as a function of the decoherence density $p$.

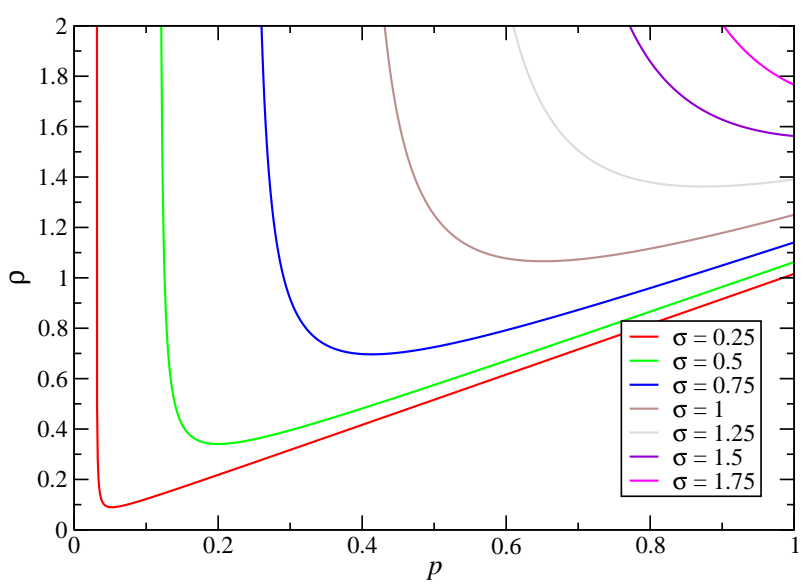

FIG. 2: (Color online) The resistivity $\rho$ as a function of the density of decoherence events $p\left(=1 / L_{\phi}\right.$, the inverse coherence length) for various disorder strengths $\sigma$. For $p<p^{*}$ ( $p^{*}$ are the poles in this graph) no finite conductivity exists. Parameters: Electron energy $E=0$, broadening $\eta=1$.

The radius of convergence $q^{*}$ is given by the singularity of $\rho$ which is nearest to the origin. I.e. $q^{*}$ is the smallest of the roots of $N_{1}$, which fulfill the relation

$$
g(q) \equiv \frac{q}{1-q^{2}} \sigma^{2}+\frac{q}{(1+q)^{2}} E^{2}=1
$$

We see that the singularities lie on an ellipse in the $\sigma-E$ plane, the semiaxes of which are determined by $q$. 
Both summands of $g(q)$ are strictly increasing for $q \in$ $[0,1)$, furthermore $g(0)=0$ and $\lim _{q \rightarrow 1} g(q)=\infty$. Thus Eq. (19) obviously has a single, real solution $q^{*} \in(0,1)$. The two other solutions are complex and fulfill $|q|>1$, hence they have no physical meaning. This guarantees that for any disorder and any electron energy there is a critical decoherence density $p^{*}$ above which ohmic behavior is achieved.

The solutions $q_{k}$ of Eq. (19) read

$$
\begin{aligned}
q_{k} & =\frac{1}{3}\left(E^{2}-\sigma^{2}-1\right) \\
& +\frac{1}{3} 2^{\frac{1}{3}} \mathrm{e}^{\mathrm{i} \frac{2}{3} k \pi} f_{1}(E, \sigma)\left(f_{2}(E, \sigma)+\sqrt{27 f_{3}(E, \sigma)}\right)^{-\frac{1}{3}} \\
& -\frac{1}{3} 2^{-\frac{1}{3}} \mathrm{e}^{-\mathrm{i} \frac{2}{3} k \pi}\left(f_{2}(E, \sigma)+\sqrt{27 f_{3}(E, \sigma)}\right)^{\frac{1}{3}}
\end{aligned}
$$

where the functions

$$
\begin{aligned}
f_{1}(E, \sigma) & =-4+5 E^{2}-E^{4}+\sigma^{2}+2 E^{2} \sigma^{2}-\sigma^{4} \\
f_{2}(E, \sigma) & =-16-24 E^{2}+15 E^{4}-2 E^{6}+6 \sigma^{2}-12 E^{2} \sigma^{2} \\
& +6 E^{4} \sigma^{2}-3 \sigma^{4}-6 E^{2} \sigma^{4}+2 \sigma^{6} \\
f_{3}(E, \sigma) & =64 E^{2}-48 E^{4}+12 E^{6}-E^{8}-4 \sigma^{4}+20 E^{2} \sigma^{4} \\
& +2 E^{4} \sigma^{4}-\sigma^{8}
\end{aligned}
$$

are polynomials and $k=0,1,2$. Note that by $\sqrt{ } \cdot$ and $(\cdot)^{\frac{1}{3}}$ we denote the solution of $z^{2}=\cdot$ and $z^{3}=\cdot$ with the smallest complex argument $\arg (z) \in[0,2 \pi)$, respectively. Using 20 we can write

$$
q^{*}=\min _{k}\left\{\left|q_{k}\right|\right\}=1-p^{*} .
$$

The dependence of $p^{*}$ on $E$ is displayed in Fig. 3 .

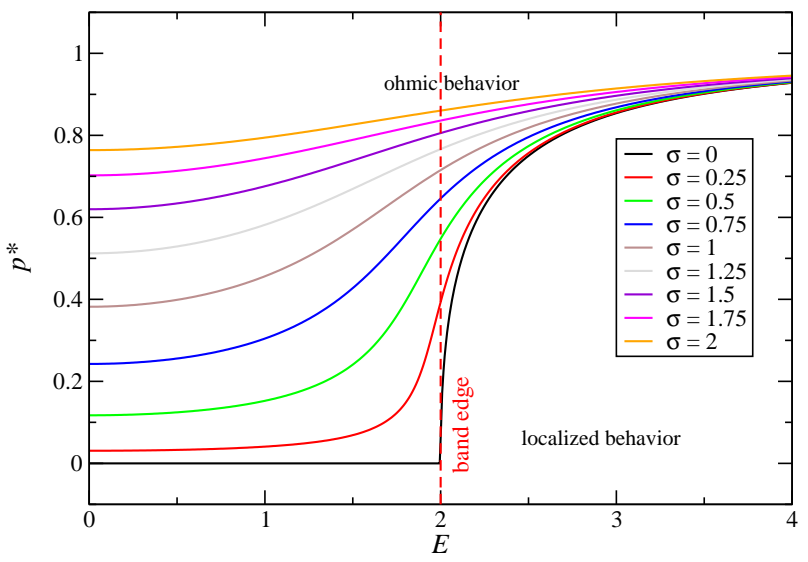

FIG. 3: (Color online) The critical decoherence density $p^{*}$ for various values of $\sigma$ as a function of the electron energy $E$. For $p>p^{*}$, ohmic behavior is achieved, whereas for $p<p^{*}$ the resistance increases exponentially with the system size. In the limit $\sigma \rightarrow 0$ we observe a kink at $E=2$, the band edge of the system without disorder.

For the interpretation of Fig. 3 note that the range $E \in[-2,2]$ is the energy band of the system without disorder. Thus only for $|E| \leq 2$ there are eigenstates, which in this case are extended states. Electrons with $|E|>2$ effectively have to tunnel through the system. This only leads to a finite resistivity if the tunneling length is reduced by increasing the decoherence parameter $p$. The physical picture is similar for the case with disorder. Now there are no extended states, and electron transmission is exponentially suppressed for any $E$. Thus $p^{*}>0$ for all values of $E$.

\section{THE GENERALIZED LYAPUNOV EXPONENT $\xi^{-1}$}

The generalized Lyapunov exponents (GLEs) are defined as 15,17

$$
L(r) \equiv \frac{1}{r} \lim _{j \rightarrow \infty} \frac{1}{j} \ln \left\langle T_{j}^{-\frac{r}{2}}\right\rangle .
$$

E.g. the second-order GLE $\xi^{-1} \equiv 2 L(r=2)$ reads

$$
\xi^{-1}=\lim _{j \rightarrow \infty} \frac{1}{j} \ln \left\langle\frac{1}{T_{j}}\right\rangle .
$$

Since, according to Eq. $10 p, \rho / p^{2}$ is the generating function of the $\left\langle 1 / T_{j}\right\rangle$, its pole $q^{*}$ determines 20 the asymptotic behavior of $\left\langle 1 / T_{j}\right\rangle$, and hence also $\xi^{-1}$ :

$$
\lim _{j \rightarrow \infty}\left\langle\frac{1}{T_{j}}\right\rangle \sim\left(\frac{1}{q^{*}}\right)^{j} \Rightarrow \xi^{-1}=-\ln \left(q^{*}\right) .
$$

Therefore $\xi^{-1}$ can be determined exactly for any set of parameters $(\sigma, E)$ using Eqs. 20p-21) and (24).

Note that apart from the energy, $\xi^{-1}$ only depends on the second moment $\sigma^{2}$ of the distribution of the onsite energies. Higher moments do not enter.

Fig. 4 displays the dependence of $\xi^{-1}$ on the disorder strength $\sigma$ for various energies $E$.

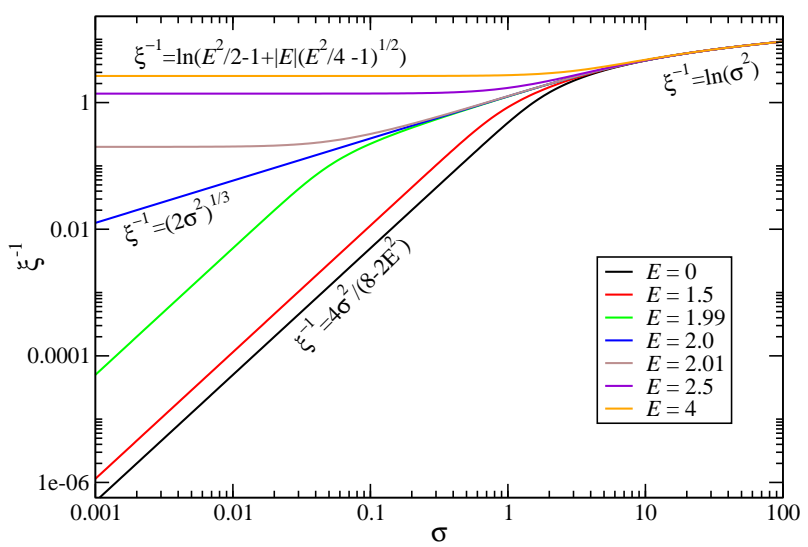

FIG. 4: (Color online) Dependence of $\xi^{-1}$ on $\sigma$ for different values of $E$.

We observe that, depending on $E$ and the disorder strength, $\xi^{-1}$ assumes different functional dependencies 
on $\sigma$. In the weak disorder limit $\sigma \rightarrow 0, \xi^{-1}$ approximates

$$
\xi^{-1}= \begin{cases}\frac{4 \sigma^{2}}{8-2 E^{2}}, & |E|<2 \\ \left(2 \sigma^{2}\right)^{\frac{1}{3}}, & |E|=2 \\ -\ln \left(1-\frac{E^{2}}{2}+\frac{|E|}{2} \sqrt{E^{2}-4}\right), & |E|>2\end{cases}
$$

and for strong disorder $\sigma \rightarrow \infty$

$$
\xi^{-1}=\ln \left(\sigma^{2}\right)
$$

as can be seen easily by inserting $z=\exp \left(-\xi^{-1}\right)$ into Eq. (17).

Outside the band $|E|>2, \xi^{-1}$ is finite also for $\sigma=0$. This is why in Fig. 4 and Eq. 25) no $\sigma$-dependence is given outside the band for weak disorder.

Using $\xi^{-1}$, we can restate the condition $q<q^{*}$ for a finite conductivity as:

$$
L_{\phi}<\frac{1}{1-\exp \left(-\xi^{-1}\right)}
$$

which for weak disorder inside the band becomes

$$
L_{\phi}<\xi
$$

As $\xi^{-1}$ is a property of the infinite coherent system, it is determined purely by $E$ and $\sigma^{2}$ and does not depend on the decoherence parameters $p$ and $\eta$. Yet via Eq. (27), the second-order GLE $\xi^{-1}$ determines the maximum coherence length for which a finite conductivity can be reached.

\section{GENERALIZED LYAPUNOV EXPONENTS AND LOCALIZATION LENGTH}

The localization length $\lambda$ is defined as the typical length scale on which the eigenstates of a disordered system decay. ${ }^{21}$ Its inverse (the standard Lyapunov exponent LE) is related to the dimensionless conductance of a coherent system via

$$
\lambda^{-1}=-\lim _{j \rightarrow \infty} \frac{1}{2 j}\left\langle\ln T_{j}\right\rangle .
$$

It is easy to see that the localization length and the GLEs are connected by

$$
\lambda^{-1}=\lim _{r \rightarrow 0} L(r) .
$$

With our method we can calculate $L(r)$ only for even $r \neq 0$, so that the limit $r \rightarrow 0$, Eq. (30), is not obvious. It remains to be investigated in the future.

We can, however, use our results to calculate $\lambda$ approximately and gain new insight into the assumptions of single parameter scaling.

One must distinguish two assumptions, which together define what is known as single parameter scaling (SPS) in localization theory! $19222 / 23$
Assumption $\mathrm{A}$ is that the variable $u_{j}=-\ln T_{j}$ obeys the central limit theorem and approaches a Gaussian distribution as $j \rightarrow \infty$.

Second, assumption B states that a single parameter suffices to characterize the Gaussian distribution, in other words, the mean $C_{1}$ and the variance $C_{2}$ are proportional to each other and that the proportionality factor is 2 ,

$$
C_{2} / C_{1}=2 \text { for SPS. }
$$

Assumptions A and B were proven to hold for the continuous Anderson model for uncorrelated weak disorder, provided complete phase randomization takes place between scattering events. $22 \mid 23$

For the discrete Anderson model, however, already the weaker assumption A was shown to be wrong at the band edges and the band center.24 Nevertheless, for weak disorder the correct power law dependencies of $\lambda^{-1}$ on $\sigma$ are reproduced, if assumption A is applied, see Eqs. 44 and (45). Only the prefactors are wrong. For $E$-values in between the band center and the band edges, assumption A even leads to the correct $\lambda^{-1}$ in leading order of $\sigma$, see Eq. 41.

In the following we adopt assumption A as an approximation and investigate how strongly assumption B is violated. We find strong violation of assumption $B$ at the band edges and at the band center. However, if for $E$-values in between and weak disorder, one makes the assumption A, then assumption B is automatically fulfilled.

The assumed Gaussian limiting distribution of the variable $u_{j}=-\ln T_{j}$ is characterized by its mean $C_{1}$ and variance $C_{2}$, where

$$
\lim _{j \rightarrow \infty} \frac{C_{1}}{j}=2 \lambda^{-1} .
$$

This means that the probability $P\left(u_{j}\right)$ to find $u_{j} \in[u, u+$ $\mathrm{d} u$ ] fulfills, for a finite system of length $j$,

$$
\lim _{j \rightarrow \infty}\left(P\left(u_{j}\right) \mathrm{d} u_{j}-\frac{1}{\sqrt{2 \pi C_{2}}} \mathrm{e}^{-\frac{\left(u_{j}-C_{1}\right)^{2}}{2 C_{2}}} \mathrm{~d} u_{j}\right)=0,
$$

which allows to relate

$$
\begin{aligned}
\xi^{-1} & =\lim _{j \rightarrow \infty} \frac{1}{j} \ln \overline{\mathrm{e}^{u_{j}}}=\lim _{j \rightarrow \infty} \frac{1}{j} \ln \left[\mathrm{e}^{C_{1}+C_{2} / 2}\right] \\
& =\lim _{j \rightarrow \infty} \frac{1}{j}\left(C_{1}+C_{2} / 2\right)
\end{aligned}
$$

where $\overline{(\cdot)}$ denotes the average $\int \mathrm{d} u_{j} P\left(u_{j}\right)(\cdot)$.

Analogously to $\xi^{-1}$, cf. Eq. 24, we can also calculate the fourth-order GLE $\chi^{-1}=4 L(4)$ (and similarly, all higher even-order GLEs),

$$
\chi^{-1} \equiv \lim _{j \rightarrow \infty} \frac{1}{j} \ln \left\langle\frac{1}{T_{j}^{2}}\right\rangle=-\ln \left(z^{*}\right)
$$

by determining $z^{*}$, the pole of the generating function $\mathcal{T}_{-2}(z)$ of $\left\langle 1 / T_{j}^{2}\right\rangle$ with the smallest absolute value. 
The definition and derivation of $\mathcal{T}_{-2}(z)$ can be found in the Appendix B. Its denominator reads

$$
N_{1} \times N_{2} \times(z-1),
$$

where $N_{1}$ is given in Eq. (17) and $N_{2}$ is a fifth-order polynomial in $z$ given in Eq. (B29).

Note that, as $1 / T_{j}^{2}$ contains up to fourth powers of the polynomials $r_{j}$ and $s_{j}$, the roots of $N_{2}$, and therefore $\chi$ itself, depend also on the third and fourth moments of the probability distribution $w(\epsilon)$ of the onsite energies. At the same time, $w(\epsilon)$ only enters through its moments into $\chi$, therefore any distributions with the same second, third and fourth moments has the same $\chi$, independently of the exact shape of the distribution. Analogously $L(6)$ depends on the first six moments of $w(\epsilon)$, etc.

As $N_{2}$ is a fifth-order polynomial, the value of $\chi$ can be calculated within numerical accuracy. Only for special cases (e.g. for $E=0$, see below in Eq. 43), $\chi$ can be represented in a closed form.

Like $\xi^{-1}$ we can relate $\chi^{-1}$ to the cumulants $C_{2}$ and $C_{1}$ under the assumption $\mathrm{A}$ :

$$
\begin{aligned}
\chi^{-1} & =\lim _{j \rightarrow \infty} \frac{1}{j} \ln \overline{\mathrm{e}^{2 u_{j}}} \\
& =\lim _{j \rightarrow \infty} \frac{1}{j}\left(2 C_{1}+2 C_{2}\right) .
\end{aligned}
$$

This equation together with Eqs. (32) and (34) leads to the localization length

$$
\lambda^{-1}=\xi^{-1}-\frac{1}{4} \chi^{-1} \quad \text { under assumption A. }
$$
lants:

We solve Eqs. (34) and (37) for the ratio of the cumu-

$$
\frac{C_{2}}{C_{1}}=\frac{2 \chi^{-1}-4 \xi^{-1}}{4 \xi^{-1}-\chi^{-1}} .
$$

We can compare this with the relation predicted by SPS theory, Eq. 31, by examining the parameter

$$
\delta=\frac{\left|\frac{C_{2}}{C_{1}}-2\right|}{2}
$$

which measures the relative deviation from SPS. Fig. 5 displays contour lines $\delta(\sigma, E)=$ const. for Gaussian onsite disorder, i.e. $\nu^{3}=0, \kappa^{4}=3 \sigma^{4}$.

The interpretation of Fig. 5 is the following. For disorder strengths $\sigma$ below the contour lines $\sigma<\sigma_{\delta}(E)$, the relative deviation from the SPS hypothesis is less than $\delta$. E.g. all points $(\sigma, E)$ below the black line in Fig. 5 fulfill $\delta<10^{-4}$.

For weak disorder and energies $0<|E|<2$, we find $\chi^{-1}=3 \xi^{-1}$ which according to Eq. 39 leads to SPS $\left(C_{2} / C_{1}=2\right)$. Using Eq. 38 , we get

$$
\lambda^{-1}=\frac{\sigma^{2}}{8-2 E^{2}}, \quad \text { for } 0<|E|<2 \text { and } \sigma \rightarrow 0,
$$

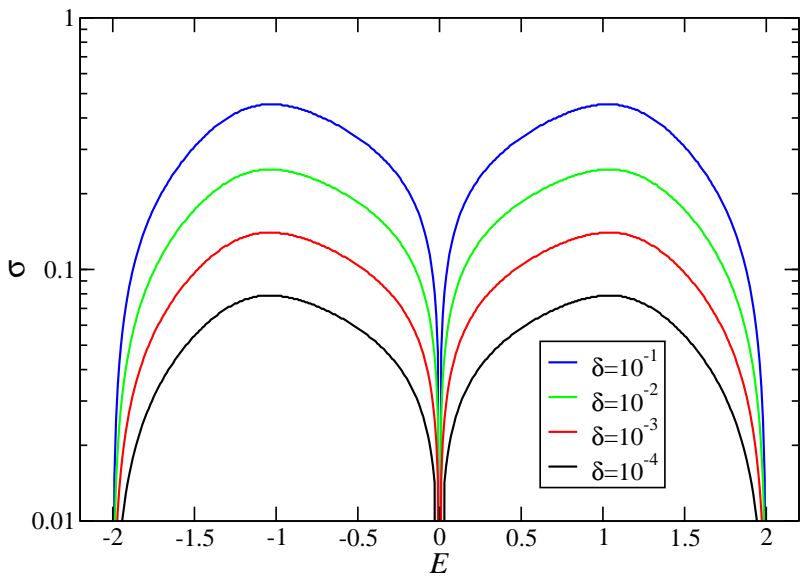

FIG. 5: (Color online) Contour lines for $\delta=\left(C_{2} / C_{1}-2\right) / 2$ which indicate the deviation from SPS. Assumed was a Gaussian onsite energy disorder.

in agreement with the well-known formula by Thouless 21 which, in this energy range, is correct also for the discrete Anderson model!25

At the band center $E=0$ we find that SPS is violated even in the limit $\sigma \rightarrow 0$. Inserting

$$
\xi^{-1}=\ln \left[\frac{\sigma^{2}}{2}+\sqrt{1+\left(\frac{\sigma^{2}}{2}\right)^{2}}\right], \quad \text { for } E=0
$$

as well as, for Gaussian onsite energy disorder $\kappa^{4}=3 \sigma^{4}$,

$$
\chi^{-1}=\ln \left[1+\frac{3}{2} \sigma^{4}+\sigma^{2} \sqrt{3+\left(\frac{3}{2} \sigma^{2}\right)^{2}}\right], \quad \text { for } E=0
$$

into Eq. (38) leads to the localization length

$$
\lambda^{-1}=\frac{2-\sqrt{3}}{4} \sigma^{2}, \quad \text { for } E=0 \text { and } \sigma \rightarrow 0 .
$$

This equation shows the correct power-law behavior $\lambda^{-1} \sim \sigma^{2}$ but deviates from the correct prefactor. 25126 This suggests that at the band center the assumption A breaks down and the simple approximation (38) between the localization length and the GLEs $\xi^{-1}$ and $\chi^{-1}$ is no longer valid. This is a signature of the band center anomaly! 24

Similarly, at the band edges $|E|=2$, SPS is violated also for $\sigma \rightarrow 0$. Eq. (38) leads to

$$
\lambda^{-1}=\left(2^{1 / 3}-\frac{1}{4} 42^{1 / 3}\right) \times \sigma^{2 / 3}, \quad \text { for }|E|=2 \text { and } \sigma \rightarrow 0,
$$

again showing the correct power-law behavior $\lambda^{-1} \sim \sigma^{2 / 3}$ but deviating from the correct prefactor for the same reason as above. 
Outside the band $|E|>2$, examining $\delta$ does not lead to agreement with SPS for any disorder strength.

In other words, if assumption A is applied as an approximation to the discrete Anderson model one obtains the correct localization length in leading order of $\sigma$ as long as $E$ is in between the band edges and the band center. Then, assumption A automatically leads to fulfillment of assumption B.

\section{CONCLUSIONS}

The present article deals with the discrete-space 1D Anderson model with uncorrelated diagonal disorder, characterized by its finite second through fourth moments $\sigma^{2}, \nu^{3}$ and $\kappa^{4}$.

When subjected to a statistical model for the effects of decoherence (parameters $p=1 / L_{\phi}$, the inverse coherence length, and $\eta$, the energy broadening due to decoherence) ${ }^{7}$ the length-dependent resistance at infinitesimal bias voltage undergoes a decoherenceinduced transition from localized, exponentially increasing to ohmic, linearly increasing behavior, i.e. we find decoherence-induced conductivity.

For the ohmic regime we derive the exact value of the resistivity $\rho$ which for given model parameters $p$ and $\eta$ only depends on the electron energy $E$ and the second moment $\sigma^{2}$ of the onsite energy disorder. Higher moments do not enter.

The critical decoherence density $p^{*}$, at which the transition from localized to ohmic behavior takes place, is connected to the second-order generalized Lyapunov exponent (GLE) $\xi^{-1}=-\ln \left(1-p^{*}\right)$.

Therefore we can state the condition for finite conductivity as $L_{\phi}<\left(1-\exp \left(-\xi^{-1}\right)\right)^{-1}$, which for weak disorder and energies inside the band becomes $L_{\phi}<\xi$, i.e. that the phase coherence length be smaller than the length $\xi$. In this way, a property of the completely coherent Anderson model $(\xi)$ determines the limits $\left(p^{*}\right)$ of the ohmic behavior in the Anderson model including decoherence.

The GLE $\xi^{-1}$ describes the exponential increase of $\left\langle 1 / T_{j}\right\rangle$, the disorder-averaged dimensionless resistance. Using the generating function of the $\left\langle 1 / T_{j}\right\rangle$ (which is nothing but $\rho / p^{2}$ ) we derive the exact value of $\xi^{-1}$ for arbitrary electron energy and disorder.

Analogously to $\xi^{-1}$, the fourth-order GLE $\chi^{-1}$ is calculated from the generating function of $\left\langle 1 / T_{j}^{2}\right\rangle$. In a similar way, all higher even-order GLEs can be determined.

Under the assumption of a Gaussian limiting distribution for $\ln T$ (assumption A), $\xi$ and $\chi$ together allow to determine the localization length $\lambda$ and the ratio of the first two cumulants of $\ln T, C_{2} / C_{1}$ approximately. By studying this ratio we conclude that the single parameter scaling (SPS) hypothesis which states $C_{2} / C_{1}=2$ is automatically fulfilled in weak disorder for energies in between the band center and the band edges. Furthermore in this energy range assumption A leads to the cor- rect weak disorder limit of the localization length. For this energy range, we present a maximum disorder $\sigma_{\delta}(E)$ which yields agreement with SPS, given a tolerance $\delta$ of deviation.

For the energies $E=0$ and $|E|=2$ (band edges and band center of the pure system), even for weak disorder the SPS hypothesis is not fulfilled. Furthermore, the localization length one obtains under assumption A does not agree with the literature results .25126 We conclude that at these energies assumption A breaks down, in accordance with Ref. 24 .

\section{Acknowledgments}

This work was supported by Deutsche Forschungsgemeinschaft under grants Nos. GRK 1240 "nanotronics" and SPP 1386 "nanostructured thermoelectric materials". O.U. acknowledges the support of the Alexander von Humboldt Foundation and the János Bolyai Research Foundation of the Hungarian Academy of Sciences. M.W. wishes to thank Ludger Santen for the support by Saarland University.

\section{Appendix A: Derivation of the recursive formula for the Green function element $G_{1 j}$}

In this section we derive Eqs. (5) and (6) which are necessary for the calculation of the transmission function. The Green function of the finite chain with wideband (virtual) contacts is the inverse of a tridiagonal, symmetric matrix:

$$
G^{-1}=\left(\begin{array}{ccccc}
E-\epsilon_{1}+\mathrm{i} \eta & -1 & 0 & \cdots & 0 \\
-1 & E-\epsilon_{2} & -1 & 0 & \vdots \\
0 & -1 & \ddots & & \\
\vdots & & & & -1 \\
0 & \ldots & 0 & -1 & E-\epsilon_{j}+\mathrm{i} \eta
\end{array}\right)
$$

As such, its components are easily derived by LU decomposition,

$$
\begin{aligned}
G^{-1} & =\left(\begin{array}{cccc}
\alpha_{1} & -1 & 0 & \ldots \\
-1 & \ddots & & \\
0 & & \ddots & -1 \\
\vdots & & -1 & \alpha_{j}
\end{array}\right) \\
& =\underbrace{\left(\begin{array}{cccc}
1 & 0 & \ldots & \\
l_{1} & 1 & \ddots & \\
0 & \ddots & \ddots & 0 \\
\vdots & 0 & l_{j-1} & 1
\end{array}\right)}_{L} \underbrace{\left(\begin{array}{cccc}
m_{1} & -1 & 0 & \ldots \\
0 & m_{2} & \ddots & \\
\vdots & \ddots & \ddots & -1 \\
\ldots & 0 & m_{j}
\end{array}\right)}_{U},
\end{aligned}
$$


where $\alpha_{i}=E-\epsilon_{i}+\mathrm{i} \delta_{i 1} \eta+\mathrm{i} \delta_{i j} \eta$ for $i=1, \ldots, j, l_{i}=-\frac{1}{m_{i}}$ for $i=1, \ldots, j-1, m_{1}=\alpha_{1}$ and $m_{i}=\alpha_{i}+l_{i-1}$ for $i=2, \ldots, j$.

From

$$
G^{-1} G=L U G=1
$$

follows that $G_{1 j}$ is the first element of the vector $x$ that fulfills

$$
L \underbrace{U x}_{y}=\delta_{i j} .
$$

Forward substitution yields

$$
y=\delta_{i j}
$$

and subsequent backward substitution in

$$
U x=\delta_{i j}
$$

gives

$$
G_{1 j}=x_{1}=\frac{1}{m_{1} \times m_{2} \times \cdots \times m_{j}} .
$$

Inserting the definitions of the $m_{j}$ we find the recursive formula

$$
G_{1 j}=\frac{1}{P_{j}}
$$

with the polynomials

$$
\begin{aligned}
& P_{0}=1 \\
& P_{1}=E-\epsilon_{1}+\mathrm{i} \eta \\
& P_{i}=\left(E-\epsilon_{i}\right) P_{i-1}-P_{i-2}, \quad i=2, \ldots, j-1 \\
& P_{j}=\left(E-\epsilon_{j}+\mathrm{i} \eta\right) P_{j-1}-P_{j-2} .
\end{aligned}
$$

As $E$, the $\epsilon_{i}$, and $\eta$ are real quantities,

$$
P_{j}=r_{j}-\eta^{2} s_{j-1}+\mathrm{i} \eta\left(s_{j}+r_{j-1}\right)
$$

is the subdivision of the polynomial $P_{j}$ into its real and imaginary parts, using the iterative polynomials $r_{i}$ and $s_{i}$ defined in (6). With the definition of the transmission function (Eq. (4)) follows Eq. (5).

\section{Appendix B: Derivation of the generating function $\mathcal{T}_{-2}$}

Using the relation (5) of the transmission function to the recursively defined polynomials $r_{j}$ and $s_{j}$ (6) we de- rive a recursive expression for $\left\langle 1 / T_{j}^{2}\right\rangle$ :

$$
\begin{aligned}
\left\langle\frac{1}{T_{j}^{2}}\right\rangle & =\frac{1}{16 \eta^{4}} \int_{-\infty}^{\infty} \mathrm{d} \epsilon_{1} \ldots \mathrm{d} \epsilon_{j}\left[\prod_{i=1}^{j} w\left(\epsilon_{i}\right)\right] \\
& \times\left(r_{j}^{2}+\eta^{2} r_{j-1}^{2}+\eta^{2} s_{j}^{2}+\eta^{4} s_{j-1}^{2}+2 \eta^{2}\right)^{2} \\
& =\frac{1}{4}+\frac{1}{16}\left(\eta^{-4} R_{j}^{4}+2 R_{j-1}^{4}+\eta^{4} R_{j-2}^{4}\right) \\
& +\frac{1}{8}\left(\eta^{-2} R_{j}^{22}+\eta^{2} R_{j-1}^{22}\right) \\
& +\frac{1}{4}\left(\eta^{-2} R_{j}+2 R_{j-1}+\eta^{2} R_{j-2}\right) \\
& +\frac{1}{8}\left(\eta^{-2} S_{j j}^{22}+\eta^{2} S_{j-1 j-1}^{22}\right) \\
& +\frac{1}{8}\left(S_{j j-1}^{22}+S_{j-1 j}^{22}\right)
\end{aligned}
$$

where the $R_{j}$ are defined in $(12)$, and

$$
\begin{aligned}
R_{j}^{4} & \equiv \mathbf{I}_{j}\left[r_{j}^{4}\right], \\
R_{j}^{22} & \equiv \mathbf{I}_{j}\left[r_{j}^{2} r_{j-1}^{2}\right] \\
& =\mathbf{I}_{j+1}\left[s_{j+1}^{2} s_{j}^{2}\right], \\
S_{j k}^{22} & \equiv \mathbf{I}_{\max \{j, k\}}\left[r_{j}^{2} s_{k}^{2}\right]
\end{aligned}
$$

using the short-hand notation for the integration

$$
\mathbf{I}_{j}[\cdot] \equiv \int_{-\infty}^{\infty} \mathrm{d} \epsilon_{1} \ldots \mathrm{d} \epsilon_{j}\left[\prod_{i=1}^{j} w\left(\epsilon_{i}\right)\right][\cdot] .
$$

Analogously we define

$$
\begin{aligned}
R_{j}^{31} & \equiv \mathbf{I}_{j}\left[r_{j}^{3} r_{j-1}\right] \\
R_{j}^{13} & \equiv \mathbf{I}_{j}\left[r_{j} r_{j-1}^{3}\right]
\end{aligned}
$$

and

$$
\begin{aligned}
S_{j k}^{211} & \equiv \mathbf{I}_{\max \{j, k\}}\left[r_{j}^{2} s_{k} s_{k-1}\right] \\
S_{j k}^{112} & \equiv \mathbf{I}_{\max \{j, k\}}\left[r_{j} r_{j-1} s_{k}^{2}\right] \\
S_{j}^{1111} & \equiv \mathbf{I}_{j}\left[r_{j} r_{j-1} s_{j} s_{j-1}\right]
\end{aligned}
$$

By (6) the $R$ and $S$ fulfill the following recursions

$$
\begin{aligned}
R_{j}^{4} & =X_{4} R_{j-1}^{4}-4 X_{3} R_{j-1}^{31}+6 X_{2} R_{j-1}^{22} \\
& -4 X_{1} R_{j-1}^{13}+R_{j-2}^{4} \\
R_{j}^{31} & =X_{3} R_{j-1}^{4}-3 X_{2} R_{j-1}^{31}+3 X_{1} R_{j-1}^{22}-R_{j-1}^{13} \\
R_{j}^{22} & =X_{2} R_{j-1}^{4}-2 X_{1} R_{j-1}^{31}+R_{j-1}^{22} \\
R^{13} & =X_{1} R_{j-1}^{4}-R_{j-1}^{31} \\
S_{j j}^{22} & =X_{4} S_{j-1 j-1}^{22}+4 X_{2} S_{j-1}^{1111}+S_{j-2 j-2}^{22} \\
& -2 X_{3}\left(S_{j-1 j-1}^{211}+S_{j-1 j-1}^{112}\right) \\
& +X_{2}\left(S_{j-1 j-2}^{22}+S_{j-2 j-1}^{22}\right) \\
& -2 X_{1}\left(S_{j-1 j-2}^{112}+S_{j-2 j-1}^{211}\right) \\
S_{j j-1}^{22} & =X_{2} S_{j-1 j-1}^{22}-2 X_{1} S_{j-1 j-1}^{112}+S_{j-2 j-1}^{22}
\end{aligned}
$$




$$
\begin{aligned}
S_{j-1 j}^{22} & =X_{2} S_{j-1 j-1}^{22}-2 X_{1} S_{j-1 j-1}^{211}+S_{j-1 j-2}^{22} \\
S_{j j}^{211} & =X_{3} S_{j-1 j-1}^{22}-2 X_{2} S_{j-1 j-1}^{112}+X_{1} S_{j-2 j-1}^{22} \\
& -X_{2} S_{j-1 j-1}^{211}+2 X_{1} S_{j-1}^{1111}-S_{j-2 j-1}^{211} \\
S_{j j}^{112} & =X_{3} S_{j-1 j-1}^{22}-2 X_{2} S_{j-1 j-1}^{211}+X_{1} S_{j-1 j-2}^{22} \\
& -X_{2} S_{j-1 j-1}^{112}+2 X_{1} S_{j-1}^{1111}-S_{j-1 j-2}^{112} \\
S_{j-1 j}^{211} & =X_{1} S_{j-1 j-1}^{22}-S_{j-1 j-1}^{211} \\
S_{j j-1}^{112} & =X_{1} S_{j-1 j-1}^{22}-S_{j-1 j-1}^{112} \\
S_{j}^{1111} & =X_{2} S_{j-1 j-1}^{22}-X_{1}\left(S_{j-1 j-1}^{211}+S_{j-1 j-1}^{112}\right)+S_{j-1}^{1111}
\end{aligned}
$$

where we have defined

$$
\begin{aligned}
X_{n} & =\mathbf{I}_{1}\left[\left(E-\epsilon_{1}\right)^{n}\right] \\
& = \begin{cases}E^{4}+6 E^{2} \sigma^{2}-4 E \nu^{3}+\kappa^{4} & \text { for } n=4 \\
E^{3}+3 E \sigma^{2}-\nu^{3} & \text { for } n=3 \\
E^{2}+\sigma^{2} & \text { for } n=2 \\
E & \text { for } n=1\end{cases}
\end{aligned}
$$

For the recursions, the following initial conditions apply:

$$
\begin{aligned}
R_{1}^{4} & =X_{4}, \quad R_{0}^{4}=1 \\
R_{-1}^{4} & =R_{0}^{31}=R_{0}^{22}=R_{0}^{13}=0 \\
S_{11}^{22} & =X_{2}, \quad S_{11}^{112}=X_{1}, \quad S_{01}^{22}=1 \\
S_{10}^{22} & =S_{11}^{211}=S_{01}^{211}=S_{10}^{112}=S_{1}^{1111}=0
\end{aligned}
$$

With this we can determine the generating functions of the $R$ and $S$ :

$$
\begin{aligned}
\mathcal{R}^{4}(z) & \equiv \sum_{j=1}^{\infty} R_{j}^{4} z^{j-1} \\
& =X_{4}+z X_{4} \mathcal{R}^{4}(z)+z+z^{2} \mathcal{R}^{4}(z) \\
& -4 z X_{3} \mathcal{R}^{31}(z)+6 z X_{2} \mathcal{R}^{22}(z)-4 z X_{1} \mathcal{R}^{13}(z) \\
\mathcal{R}^{31}(z) & \equiv \sum_{j=1}^{\infty} R_{j}^{31} z^{j-1} \\
& =X_{3}+z X_{3} \mathcal{R}^{4}(z)-3 z X_{2} \mathcal{R}^{31}(z) \\
& +3 z X_{1} \mathcal{R}^{22}(z)-z \mathcal{R}^{13}(z) \\
\mathcal{R}^{22}(z) & \equiv \sum_{j=1}^{\infty} R_{j}^{22} z^{j-1} \\
& =X_{2}+z X_{2} \mathcal{R}^{4}(z)-2 z X_{1} \mathcal{R}^{31}(z)+z \mathcal{R}^{22}(z) \\
\mathcal{R}^{13}(z) & \equiv \sum_{j=1}^{\infty} R_{j}^{13} z^{j-1} \\
& =X_{1}+z X_{1} \mathcal{R}^{4}(z)-z \mathcal{R}^{31}(z)
\end{aligned}
$$

$$
\begin{aligned}
\mathcal{S}_{11}^{22}(z) & \equiv \sum_{j=1}^{\infty} S_{j j}^{22} z^{j-1} \\
& =X_{2}+z X_{4} \mathcal{S}_{11}^{22}(z)+4 z X_{2} \mathcal{S}^{1111}(z) \\
& -2 z X_{3}\left(\mathcal{S}_{11}^{211}(z)+\mathcal{S}_{11}^{112}(z)\right) \\
& +z X_{2}\left(\mathcal{S}_{10}^{22}(z)+\mathcal{S}_{01}^{22}(z)\right) \\
& -2 X_{1}\left(\mathcal{S}_{10}^{112}(z)+\mathcal{S}_{01}^{211}(z)\right)+z^{2} \mathcal{S}_{11}^{22}(z) \\
\mathcal{S}_{10}^{22}(z) & \equiv \sum_{j=1}^{\infty} S_{j j-1}^{22} z^{j-1} \\
& =z X_{2} \mathcal{S}_{11}^{22}(z)-2 z X_{1} \mathcal{S}_{11}^{112}(z)+z \mathcal{S}_{01}^{22}(z) \\
\mathcal{S}_{01}^{22}(z) & \equiv \sum_{j=1}^{\infty} S_{j-1 j}^{22} z^{j-1} \\
= & 1+z X_{2} \mathcal{S}_{11}^{22}(z)-2 z X_{1} \mathcal{S}_{11}^{211}(z)+z \mathcal{S}_{10}^{22}(z) \\
\mathcal{S}_{11}^{211}(z) \equiv & \sum_{j=1}^{\infty} S_{j j}^{211} z^{j-1} \\
= & z X_{3} \mathcal{S}_{11}^{22}(z)-2 z X_{2} \mathcal{S}_{11}^{112}(z)+z X_{1} \mathcal{S}_{01}^{22}(z) \\
& -z X_{2} \mathcal{S}_{11}^{211}(z)+2 z X_{1} \mathcal{S}^{1111}(z)-z \mathcal{S}_{01}^{211}(z) \\
\mathcal{S}_{11}^{112}(z) \equiv & \sum_{j=1}^{\infty} S_{j j}^{112} z^{j-1} \\
= & X_{1}+z X_{3} \mathcal{S}_{11}^{22}(z)-2 z X_{2} \mathcal{S}_{11}^{211}(z)+z X_{1} \mathcal{S}_{10}^{22}(z) \\
- & z X_{2} \mathcal{S}_{11}^{112}(z)+2 z X_{1} \mathcal{S}^{1111}(z)-z \mathcal{S}_{10}^{112}(z)
\end{aligned}
$$$$
\mathcal{S}_{01}^{211}(z) \equiv \sum_{j=1}^{\infty} S_{j-1 j}^{211} z^{j-1}
$$$$
=z X_{1} \mathcal{S}_{11}^{22}(z)-z \mathcal{S}_{11}^{211}(z)
$$$$
\mathcal{S}_{10}^{112}(z) \equiv \sum_{j=1}^{\infty} S_{j j-1}^{112} z^{j-1}
$$$$
=z X_{1} \mathcal{S}_{11}^{22}(z)-z \mathcal{S}_{11}^{112}(z)
$$

$$
\begin{aligned}
\mathcal{S}^{1111}(z) & \equiv \sum_{j=1}^{\infty} S_{j}^{1111} z^{j-1} \\
& =z X_{2} \mathcal{S}_{11}^{22}(z)-z X_{1}\left(\mathcal{S}_{11}^{211}(z)+\mathcal{S}_{11}^{112}(z)\right) \\
& +z \mathcal{S}^{1111}(z)
\end{aligned}
$$

Eqs. (B11)-B14 and (B15)-(B22) constitute linear systems of equations for the generating functions $\mathcal{R}$ and $\mathcal{S}$, respectively.

The solutions read

$$
\begin{aligned}
\mathcal{R}^{4}(z) & =\frac{1}{N_{2}}\left[-z^{4}+\left(-36 X_{1}^{2} X_{2}+3 X_{2}-X_{4}\right.\right. \\
& \left.+8 X_{1} X_{3}+1+6 X_{2}^{2}-6 X_{1}^{2}+24 X_{1}^{4}\right) z^{3} \\
& +\left(-6 X_{1}^{2} X_{4}+3 X_{2} X_{4}-8 X_{1} X_{3}+12 X_{1}^{2} X_{2}\right. \\
& -3 X_{2}-18 X_{2}^{3}+1-4 X_{1}^{2}+X_{4}-4 X_{3}^{2}
\end{aligned}
$$




$$
\begin{aligned}
& \left.+24 X_{1} X_{2} X_{3}\right) z^{2}+\left(-1-3 X_{2} X_{4}+4 X_{1}^{2}\right. \\
& \left.\left.+X_{4}-6 X_{2}^{2}+4 X_{3}^{2}\right) z-X_{4}\right] \\
\mathcal{R}^{22}(z) & =\frac{1}{N_{2}}\left[\left(-2 X_{1}^{2}+X_{2}\right) z^{2}+\left(2 X_{1} X_{3}-3 X_{2}^{2}\right) z\right. \\
& \left.-X_{2}\right]
\end{aligned}
$$

and

$$
\begin{aligned}
& \mathcal{S}_{11}^{22}(z)=\frac{1}{N_{2}}\left[\left(-2 X_{1}^{2}+X_{2}\right) z^{2}+\left(2 X_{1} X_{3}-3 X_{2}^{2}\right) z\right. \\
& \left.-X_{2}\right] \\
& \mathcal{S}_{10}^{22}(z)=\frac{1}{N_{1} N_{2}(z-1)}\left[-z^{8}+\left(24 X_{1}^{4}-36 X_{1}^{2} X_{2}\right.\right. \\
& \left.-4 X_{1}^{2}+8 X_{1} X_{3}+6 X_{2}^{2}-X_{4}+2 X_{2}+1\right) z^{7} \\
& +\left(-48 X_{1}^{6}+96 X_{1}^{4} X_{2}+8 X_{1}^{4}-16 X_{1}^{3} X_{3}\right. \\
& -48 X_{1}^{2} X_{2}^{2}-4 X_{1}^{2} X_{4}+4 X_{1}^{2} X_{2}+32 X_{1} X_{2} X_{3} \\
& -12 X_{2}^{3}-6 X_{1}^{2}-8 X_{1} X_{3}-4 X_{3}^{2}+2 X_{2} X_{4} \\
& \left.+2 X_{2}^{2}+X_{4}-2 X_{2}+3\right) z^{6}+\left(32 X_{1}^{6}+12 X_{1}^{4} X_{4}\right. \\
& -72 X_{1}^{4} X_{2}-48 X_{1}^{3} X_{2} X_{3}+36 X_{1}^{2} X_{2}^{3}-28 X_{1}^{4} \\
& +24 X_{1}^{3} X_{3}+8 X_{1}^{2} X_{3}^{2}-12 X_{1}^{2} X_{2} X_{4}+24 X_{1}^{2} X_{2}^{2} \\
& +24 X_{1} X_{2}^{2} X_{3}-18 X_{2}^{4}-2 X_{1}^{2} X_{4}+46 X_{1}^{2} X_{2} \\
& -12 X_{1} X_{2} X_{3}-4 X_{2} X_{3}^{2}+3 X_{2}^{2} X_{4}+2 X_{2}^{3} \\
& +14 X_{1}^{2}-8 X_{1} X_{3}+4 X_{3}^{2}-2 X_{2} X_{4}-16 X_{2}^{2} \\
& \left.+2 X_{4}-4 X_{2}-3\right) z^{5}+\left(-8 X_{1}^{4} X_{4}+8 X_{1}^{4} X_{2}\right. \\
& +32 X_{1}^{3} X_{2} X_{3}-24 X_{1}^{2} X_{2}^{3}-20 X_{1}^{4}-8 X_{1}^{3} X_{3} \\
& -12 X_{1}^{2} X_{3}^{2}+10 X_{1}^{2} X_{2} X_{4}+16 X_{1}^{2} X_{2}^{2} \\
& -4 X_{1} X_{2}^{2} X_{3}+3 X_{2}^{4}+6 X_{1}^{2} X_{4}-2 X_{1}^{2} X_{2} \\
& -28 X_{1} X_{2} X_{3}+4 X_{2} X_{3}^{2}-3 X_{2}^{2} X_{4}+14 X_{2}^{3} \\
& +8 X_{1}^{2}+8 X_{1} X_{3}+4 X_{3}^{2}-2 X_{2} X_{4}-X_{2}^{2}-2 X_{4} \\
& \left.+4 X_{2}-3\right) z^{4}+\left(16 X_{1}^{4}+4 X_{1}^{2} X_{3}^{2}-2 X_{1}^{2} X_{2} X_{4}\right. \\
& -8 X_{1}^{2} X_{2}^{2}-4 X_{1} X_{2}^{2} X_{3}+3 X_{2}^{4}+2 X_{1}^{2} X_{4} \\
& -14 X_{1}^{2} X_{2}+4 X_{1} X_{2} X_{3}-2 X_{2}^{3}-12 X_{1}^{2}-4 X_{3}^{2} \\
& \left.+2 X_{2} X_{4}+11 X_{2}^{2}-X_{4}+2 X_{2}+3\right) z^{3} \\
& +\left(-2 X_{1}^{2} X_{4}+2 X_{1}^{2} X_{2}+4 X_{1} X_{2} X_{3}-2 X_{2}^{3}\right. \\
& \left.-2 X_{1}^{2}-X_{2}^{2}+X_{4}-2 X_{2}+1\right) z^{2}+\left(2 X_{1}^{2}\right. \\
& \left.\left.-X_{2}^{2}-1\right) z\right] \\
& \mathcal{S}_{01}^{22}(z)=\frac{1}{N_{1} N_{2}(z-1)}\left[-z^{7}+\left(20 X_{1}^{4}-32 X_{1}^{2} X_{2}-4 X_{1}^{2}\right.\right. \\
& \left.+8 X_{1} X_{3}+5 X_{2}^{2}-X_{4}+2 X_{2}+1\right) z^{6}+\left(-16 X_{1}^{6}\right. \\
& +48 X_{1}^{4} X_{2}-8 X_{1}^{3} X_{3}-36 X_{1}^{2} X_{2}^{2}-4 X_{1}^{2} X_{4} \\
& +8 X_{1}^{2} X_{2}+28 X_{1} X_{2} X_{3}-10 X_{2}^{3}-4 X_{1}^{2} \\
& -8 X_{1} X_{3}-4 X_{3}^{2}+2 X_{2} X_{4}+2 X_{2}^{2}+X_{4}-2 X_{2} \\
& +3) z^{5}+\left(4 X_{1}^{4} X_{4}-16 X_{1}^{4} X_{2}-16 X_{1}^{3} X_{2} X_{3}\right. \\
& +12 X_{1}^{2} X_{2}^{3}-16 X_{1}^{4}+8 X_{1}^{3} X_{3}+4 X_{1}^{2} X_{3}^{2} \\
& -8 X_{1}^{2} X_{2} X_{4}+16 X_{1}^{2} X_{2}^{2}+20 X_{1} X_{2}^{2} X_{3}-15 X_{2}^{4}
\end{aligned}
$$

$$
\begin{aligned}
& +32 X_{1}^{2} X_{2}-12 X_{1} X_{2} X_{3}-4 X_{2} X_{3}^{2}+3 X_{2}^{2} X_{4} \\
& +2 X_{2}^{3}+12 X_{1}^{2}-8 X_{1} X_{3}+4 X_{3}^{2}-2 X_{2} X_{4} \\
& \left.-13 X_{2}^{2}+2 X_{4}-4 X_{2}-3\right) z^{4}+\left(-4 X_{1}^{4}\right. \\
& -4 X_{1}^{2} X_{3}^{2}+4 X_{1}^{2} X_{2} X_{4}+4 X_{1}^{2} X_{2}^{2}-4 X_{1} X_{2}^{2} X_{3} \\
& +3 X_{2}^{4}+4 X_{1}^{2} X_{4}-4 X_{1}^{2} X_{2}-20 X_{1} X_{2} X_{3} \\
& +4 X_{2} X_{3}^{2}-3 X_{2}^{2} X_{4}+10 X_{2}^{3}+4 X_{1}^{2}+8 X_{1} X_{3} \\
& \left.+4 X_{3}^{2}-2 X_{2} X_{4}-X_{2}^{2}-2 X_{4}+4 X_{2}-3\right) z^{3} \\
& +\left(-4 X_{1}^{2} X_{2}+4 X_{1} X_{2} X_{3}-2 X_{2}^{3}-8 X_{1}^{2}-4 X_{3}^{2}\right. \\
& \left.+2 X_{2} X_{4}+8 X_{2}^{2}-X_{4}+2 X_{2}+3\right) z^{2}+\left(-X_{2}^{2}\right. \\
& \left.\left.+X_{4}-2 X_{2}+1\right) z-1\right]
\end{aligned}
$$

where

$$
N_{1}=z^{3}+\left(1-2 X_{1}^{2}+X_{2}\right) z^{2}+\left(-1+X_{2}\right) z-1
$$

was already presented in Eq. 177 and

$$
\begin{aligned}
N_{2} & =z^{5}+\left(-8 X_{1} X_{3}+36 X_{1}^{2} X_{2}-6 X_{2}^{2}-24 X_{1}^{4}\right. \\
& \left.-3 X_{2}+6 X_{1}^{2}-1+X_{4}\right) z^{4}+\left(6 X_{1}^{2} X_{4}+3 X_{2}\right. \\
& -3 X_{2} X_{4}-2-X_{4}+18 X_{2}^{3}+8 X_{1} X_{3}+4 X_{1}^{2} \\
& \left.-24 X_{1} X_{2} X_{3}+4 X_{3}^{2}-12 X_{1}^{2} X_{2}\right) z^{3}+\left(-10 X_{1}^{2}\right. \\
& \left.+6 X_{2}^{2}-X_{4}+2-4 X_{3}^{2}+3 X_{2} X_{4}+3 X_{2}\right) z^{2} \\
& +\left(X_{4}-3 X_{2}+1\right) z-1 .
\end{aligned}
$$

Here we have only displayed those generating functions which enter into the generating function of $\left\langle 1 / T_{j}^{2}\right\rangle$ defined below. The other functions $\mathcal{R}(z)$ and $\mathcal{S}(z)$ have similar expressions, particularly the same denominators.

Using $(\mathrm{B} 2)$ and the generating functions for the $\mathcal{R}$ and $\mathcal{S}$ (B23) $(\mathrm{B} 27)$ we arrive at the generating function of $\left\langle 1 / \overline{T_{j}^{2}}\right\rangle$ :

$$
\begin{aligned}
\mathcal{T}_{-2}(z) & \equiv \sum_{j=1}^{\infty}\left\langle\frac{1}{T_{j}^{2}}\right\rangle z^{j-1} \\
& =\frac{5}{8}+\frac{1}{4} \frac{1}{1-z}+\left(\frac{\eta^{2}}{4}+\frac{\eta^{4}}{16}\right) z \\
& +\left(\frac{1}{16 \eta^{4}}+\frac{1}{8} z+\frac{\eta^{4}}{16} z^{2}\right) \mathcal{R}^{4}(z) \\
& +\left(\frac{1}{8 \eta^{2}}+\frac{\eta^{2}}{8} z\right)\left(\mathcal{R}^{22}(z)+\mathcal{S}_{11}^{22}(z)\right) \\
& +\left(\frac{1}{4 \eta^{2}}+\frac{1}{2} z+\frac{\eta^{2}}{4} z^{2}\right) \mathcal{R}(z) \\
& +\frac{1}{8}\left(\mathcal{S}_{10}^{22}(z)+\mathcal{S}_{01}^{22}(z)\right)
\end{aligned}
$$

where $\mathcal{R}(z)$ was defined in Eq. 16 . 
Appendix C: Calculation of the generating function of $\left\langle 1 / T_{j}^{2}\right\rangle$ without coupling to external leads

Using the Green function of the tight-binding Anderson Hamiltonian without coupling to the environment,

$$
G \equiv \lim _{\eta \rightarrow 0}[E-H+\mathrm{i} \eta \mathbf{1}]^{-1}
$$

1 being the unit matrix of adequate size, one defines the transmission probability of an electron between sites $|1\rangle$ and $|j\rangle$ as

$$
T_{j} \equiv|\langle 1|G| j\rangle|^{2}
$$

Similar to Eqs. (5) and (6) a recursive calculation of the transmission is possible:

$$
\frac{1}{T_{j}}=\lim _{\eta \rightarrow 0}\left(R_{j}^{2}+I_{j}^{2}\right)
$$

where the $R_{j}$ and $I_{j}$ fulfill

$$
\begin{aligned}
R_{j} & =\left(E-\epsilon_{j}\right) R_{j-1}-\eta I_{j-1}-R_{j-2}, \\
I_{j} & =\left(E-\epsilon_{j}\right) I_{j-1}+\eta R_{j-1}-I_{j-2} .
\end{aligned}
$$

Into the calculation of

$$
\mathcal{T}_{-1}(z) \equiv \sum_{j=1}^{\infty}\left\langle\frac{1}{T_{j}}\right\rangle z^{j-1}
$$

enter the 7 generating functions of the disorder averages of the two-factor products $R_{j}^{2}, R_{j} R_{j-1}, I_{j}^{2}, I_{j} I_{j-1}, R_{j} I_{j}$,
$R_{j} I_{j-1}, I_{j} R_{j-1}$. The solution reads

$$
\mathcal{T}_{-1}(z)=\frac{-z^{2}+\left(E^{2}-\sigma^{2}-1\right) z-E^{2}-\sigma^{2}}{z^{3}+\left(\sigma^{2}-E^{2}+1\right) z^{2}+\left(\sigma^{2}+E^{2}-1\right) z-1}
$$

and has the same poles as $\mathcal{R}$ in Eq. 16.

Similarly, into the calculation of

$$
\mathcal{T}_{-2}(z) \equiv \sum_{j=1}^{\infty}\left\langle\frac{1}{T_{j}^{2}}\right\rangle z^{j-1}
$$

enter the 30 generating functions of the disorder averages of all four-factor products composed of $R_{j}, I_{j}, R_{j-1}$, and $I_{j-1}$.

The closed form of $\mathcal{T}_{-2}(z)$ is a very long expression, yet its denominator reads

$$
N_{1}^{3} \times N_{2}^{5} \times(z-1)
$$

with the same $N_{1}$ and $N_{2}$ as defined above in Eqs. 177 and $\mathrm{B} 29$.

Therefore, the generating functions $\mathcal{T}_{-1}(z)$ and $\mathcal{T}_{-2}(z)$ have the same poles $q^{*}$ and $z^{*}$ as reported above, and we find the same asymptotic behavior of $\left\langle 1 / T_{j}\right\rangle$ and $\left\langle 1 / T_{j}^{2}\right\rangle$ as $j \rightarrow \infty$. Hence the generalized Lyapunov exponents $\xi^{-1}$ and $\chi^{-1}$ of an infinite system are independent of whether one takes an environment into account or not.
1 P. W. Anderson, Phys. Rev. 109, 1492 (1958).

2 A. Lagendijk, B. van Tiggelen, and D. S. Wiersma, Phys. Today 62, 24 (2009).

3 F. Evers and A. D. Mirlin, Rev. Mod. Phys. 80, 1355 (2008).

4 G. Modugno, Rep. Prog. Phys. 73, 102401 (2010).

5 V. Berezinskii, Sov. Phys.-JETP 38, 620 (1974).

${ }^{6} \mathrm{~S}$. Datta, Electronic transport in mesoscopic systems (Cambridge University Press, 1995).

7 M. Zilly, O. Ujsághy, and D. E. Wolf, Eur. Phys. J. B 68, 237 (2009).

8 M. Zilly, O. Ujsághy, and D. E. Wolf, Mater. Res. Soc. Symp. Proc. 1260, 1260-T12-02 (2010).

9 M. Zilly, Ph.D. thesis, Universität Duisburg-Essen (2010), URL http://d-nb.info/1003903363

10 B. Xu, P. Zhang, X. Li, and N. Tao, Nano Lett. 4, 1105 (2004).

11 M. Zilly, O. Ujsághy, and D. E. Wolf, Phys. Rev. B 82, 125125 (2010).

12 A. Gogolin, V. Mel'nikov, and E. Rashba, Sov. Phys.JETP 42, 168 (1976).

13 R. Hey, K. Maschke, and M. Schreiber, Phys. Rev. B 52, 8184 (1995).

14 D. L. Maslov, V. I. Yudson, A. M. Somoza, and M. Ortuño,
Phys. Rev. Lett. 102, 216601 (2009).

15 G. Paladin and A. Vulpiani, Phys. Rev. B 35, 2015 (1987).

16 R. Zillmer and A. Pikovsky, Phys. Rev. E 67, 061117 (2003).

17 E. Gurevich and A. Iomin, Phys. Rev. E 83, 011128 (2011).

18 K. Mallick and P. Marcq, Phys. Rev. E 66, 041113 (2002).

19 B. Kramer and A. MacKinnon, Rep. Prog. Phys. 56, 1469 (1993).

20 H. S. Wilf, generatingfunctionology (Academic Press, 1994).

21 D. J. Thouless, in Ill-condensed matter: Proceedings of the Les Houches Summer School, Session XXXI, 3 July18 August 1978, edited by R. Balian, R. Maynard, and G. Toulouse (North-Holland, 1979), pp. 1-62.

22 P. W. Anderson, D. J. Thouless, E. Abrahams, and D. S. Fisher, Phys. Rev. B 22, 3519 (1980).

23 B. Shapiro, Philos. Mag. B 56, 1031 (1987).

24 H. Schomerus and M. Titov, Phys. Rev. B 67, 100201 (2003).

25 M. Kappus and F. Wegner, Z. Phys. B 45, 15 (1981).

26 Derrida, B. and Gardner, E., J. Phys. France 45, 1283 (1984).

27 L. I. Deych, M. V. Erementchouk, A. A. Lisyansky, and B. L. Altshuler, Phys. Rev. Lett. 91, 096601 (2003). 
${ }^{28}$ V. E. Kravtsov and V. I. Yudson, Phys. Rev. B 82, 195120 (2010). 